\title{
Manejo da dor neonatal: influência de fatores psicológicos e organizacionais
}

\author{
Neonatal pain management: Influence of \\ psychological and organizational factors
}

\author{
Sandra Willéia MARTINS \\ Sônia Regina Fiorim ENUMO² \\ Kely Maria Pereira de PAULA ${ }^{3}$
}

\begin{abstract}
Resumo
Com enfoque preventivo de riscos ao desenvolvimento, este estudo analisou fatores que influenciam o manejo de dor em prematuros por 84 profissionais de saúde de uma unidade neonatal. Variáveis do ambiente, como clima e diagnóstico organizacional, e pessoais, como estresse, enfrentamento e crenças sobre prematuridade e dor neonatal, foram avaliados por sete instrumentos. Resultados evidenciaram que participantes associam a prematuridade ao peso de nascimento, reconhecem a importância do tratamento da dor (97\%), mas conhecem pouco sobre sua avaliação e medidas (32\%), realizando a maioria de 20 procedimentos invasivos sem analgesia (70\%). O principal estressor foi o ambiente de trabalho, descrito como caótico e requerendo mudanças, mas houve equilíbrio entre esforço e recompensa na percepção do estresse ocupacional, cujo enfrentamento era do tipo "controle". Discute-se a influência do fator organizacional no engajamento-desengajamento desses profissionais em práticas adequadas de alívio da dor, subsidiando intervenções voltadas à assistência neonatal humanizada.
\end{abstract}

Palavras-chave: Clima organizacional; Dor; Enfrentamento; Estresse; Nascimento prematuro; Profissionais da saúde.

\begin{abstract}
Focusing on preventing developmental risks, this study examined the factors influencing pain management in premature infants according to 84 health professionals in a neonatal unit. Environmental variables, such as organizational climate and diagnosis, and personal variables, such as stress, coping, and beliefs about prematurity and neonatal pain, were evaluated using seven instruments. Results showed that the participants associate prematurity with birth weight, recognize the importance of pain management (97\%), but know little about pain measurement and evaluation (32\%)

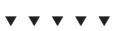

${ }^{1}$ Hospital Universitário Cassiano Antonio de Moraes, Departamento Materno Infantil, Unidade de Terapia Intensiva. Vitória, ES, Brasil.

2 Pontifícia Universidade Católica de Campinas, Centro de Ciências da Vida, Programa de Pós-Graduação em Psicologia. Av. John Boyd Dunlop, s/n., Jardim Ipaussurama, 13060-904, Campinas, SP, Brasil. Correspondência para/Correspondence to: S.R.F. ENUMO. E-mail: <sonia.enumo@puc-campinas.edu.br>.

3 Universidade Federal do Espírito Santo, Programa de Pós-Graduação em Psicologia. Vitória, ES, Brasil.

Artigo elaborado a partir da tese de S.W. MARTINS, intitulada "Manejo de dor neonatal: contexto organizacional, estresse e coping dos profissionais de saúde". Universidade Federal do Espírito Santo, 2014.

Apoio: Conselho Nacional de Desenvolvimento Científico e Tecnológico (Processo n 501079/2010-6) e Fundo de Apoio à Ciência e à Tecnologia de Vitória/Companhia de Desenvolvimento de Vitória.
} 
and most of the 20 invasive procedures are performed without analgesia (70\%). The major stressor identified was work environment, described as chaotic and requiring changes, but there was a balance between effort and reward in the perception of occupational stress, and coping was characterized by an effort to control the situation. The influence of organizational factors on the engagement-disengagement of these professionals in effective pain management practices to support interventions aimed at humanized neonatal care was discussed.

Keywords: Organizational climate; Pain; Coping behavior; Stress; Premature birth; Health professionals.

A prematuridade - nascimento antes da $37^{a}$ semana de gestação -, representa a maior causa de morbidade e mortalidade infantil no mundo, equivalendo, no Brasil, a 11,8\% dos nascimentos (Passini Junior et al., 2014). Essas crianças têm suas chances aumentadas de serem submetidas a procedimentos dolorosos desde o início da vida, durante a internação em Unidades de Terapia Intensiva Neonatal (UTIN).

Estudos da área da Neonatologia têm fornecido dados empíricos, especificamente relacionados aos efeitos em longo prazo da experiência dolorosa no início da vida, mostrando uma importante associação entre presença de dor repetitiva sem medidas analgésicas adequadas e anormalidades na maturação cerebral (Brummelte et al., 2012; McPherson et al., 2015). O Recém-Nascido Prematuro (RNPT), em especial aquele com peso de nascimento menor que 1.000 g, está mais vulnerável aos efeitos nefastos da alteração no processamento cerebral em resposta ao estresse da dor, resultando em sensibilidade dolorosa alterada, deficit de aprendizagem e distúrbios neurocomportamentais na infância e adolescência (Grunau, 2013).

Apesar do conhecimento atual, profissionais de saúde que atuam em unidades neonatais não têm adotado, com a frequência esperada, medidas de alívio de dor na realização de procedimentos invasivos de rotina, sejam farmacológicas ou não (Hall, 2012; Linhares et al., 2012, 2014). A não adesão desses profissionais às medidas de manejo de dor pode estar relacionada às seguintes variáveis: (a) falta de informação sobre avaliação e consequências da dor no neonato (Aymar, Lima, Santos, Moreno, \& Coutinho, 2014); (b) crenças sobre a falta de capacidade do RNPT para demonstrar comportamentos que expressam dor e sua sensibilidade aos procedimentos invasivos dolorosos (Anand, 634 Stevens, \& McGrath, 2007; Martins, Dias, Paula, \&
Enumo, 2013a; Ramos, Enumo, Paula, \& Vicente, 2010); (c) cultura e clima organizacional caracterizados, por exemplo, pela falta de colaboração entre os profissionais na tomada de decisão sobre manejo de dor, limitação à autonomia profissional, entre outros (Martins, Cruzeiro, Paula, \& Enumo, 2013b; Stevens et al., 2011a); (d) estressores do ambiente e da rotina, os quais repercutem diretamente na qualidade do cuidado neonatal, dificultando a adoção de medidas adequadas no alívio da dor (Theme Filha, Costa, \& Guilam, 2013); e (e) estratégias de enfrentamento ou coping do profissional da UTIN, reconhecidos como ações ou reavaliações cognitivas (controle, esquiva ou manejo), que interagem com os estressores e afetam saúde e desempenho no trabalho (Silva \& Marcolan, 2015)

As variáveis sociais e culturais (crenças, recursos e valores e demandas situacionais) podem gerar ou acentuar um quadro de estresse pessoal e ocupacional (Aldwin, 2009). Na UTIN, o profissional de saúde pode apresentar problemas de ordem emocional e desajustes no ambiente de trabalho, desencadeando sua incapacidade em atender às necessidades dos pacientes e dificuldades no relacionamento com os familiares (Montali, Monica, Riva, \& Cipriani, 2011).

No caso da adesão à prática do manejo da dor, é preciso verificar quais elementos do contexto da assistência ao RNPT esses profissionais avaliam como estressantes. A partir dessa avaliação, as pessoas apresentam estratégias de enfrentamento, entendidas pela Teoria Motivacional do Coping (TMC) como regulação do comportamento, da emoção e da orientação motivacional diante do estresse psicológico (Skinner \& Wellborn, 1994).

A Teoria Motivacional do Coping postula como eventos estressores universais tudo o que ameaça ou desafia as três necessidades psicológicas 
básicas de relacionamento, competência e autonomia (Skinner \& Wellborn, 1994). Nesse modelo, o alcance em que essas necessidades são satisfeitas determina se o indivíduo terá "engajamento" ou "desengajamento" em relação ao estressor (Ramos, Enumo, \& Paula, 2015), que poderia ser representado pelo trabalho em UTIN. Dessa forma, a questão central deste estudo está na compreensão de como ocorre o engajamento dos profissionais na assistência adequada ao RN, podendo subsidiar melhores práticas, como o manejo efetivo da dor neonatal. Se o ambiente da UTIN se apresentar ou for percebido como estruturado ou desorganizado, provavelmente haverá diferenças na ocorrência de respostas mais adaptativas.

$\mathrm{Na}$ análise desse engajamento dos profissionais de saúde, o clima organizacional é uma variável importante. É definido como uma condição temporária da atmosfera presente nas organizações, originada mediante práticas, procedimentos e recompensas percebidos diariamente pelos funcionários, intimamente ligada ao comportamento dos gestores e às ações por estes recompensadas (Menezes, Sampaio, Gomes, Teixeira, \& Santos, 2009). Um clima organizacional desfavorável poderá afetar as necessidades de competência, autonomia e relacionamento. Está atrelado à falta de integração funcionário/instituição, baixo comprometimento com a qualidade da assistência, pacientes insatisfeitos, desmotivação, pouco aproveitamento nos treinamentos e surgimento de doenças psicossomáticas (Bispo, 2006).

Em relação à necessidade de competência, perceber os estressores do ambiente como uma ameaça pode gerar nos profissionais de saúde respostas de enfrentamento menos engajadas. O desconhecimento das consequências da exposição do $\mathrm{RN}$ a procedimentos dolorosos, levando-o à condição de estresse tóxico (Shonkoff, Ricther, Gaag, \& Bhutta, 2012), por si só pode levar a um desengajamento do manejo da dor; mas, a percepção da própria ignorância sobre essas consequências também pode levar a respostas pouco adaptadas para a situação, ao ser percebida como uma ameaça à competência profissional.
Em resumo, um quadro de ameaça à autonomia e à competência profissional somado a um clima organizacional mais competitivo, que não promove a satisfação da necessidade de relacionamento no serviço, ou caótico, em termos de regras e segurança no emprego, pode favorecer um padrão de desengajamento frente a uma assistência adequada. Esse processo pode ser mediado pelas crenças dos profissionais de saúde sobre o prematuro, concepções nem sempre favoráveis ao manejo da dor (Martins et al., 2013a). Dessa maneira, diante das pesquisas nacionais que integram esses temas, este estudo pretendeu descrever e analisar como o clima organizacional da UTIN e as respostas de estresse e de enfrentamento de médicos, enfermeiros e técnicos de enfermagem, assim como suas crenças sobre prematuridade e dor neonatal, facilitam ou dificultam seu engajamento no manejo da dor em RN internados.

\section{Método}

Esta pesquisa adotou delineamento misto, com uso de abordagens qualitativa e quantitativa. Foi realizada na UTIN de um hospital público estadual terciário de urgência e emergência, com serviço materno-infantil, composto por maternidade de alto risco e enfermaria pediátrica da Grande Vitória, ES.

\section{Participantes}

A amostra de conveniência foi formada por médicos, enfermeiros e técnicos de enfermagem do quadro permanente e de designação temporária do referido hospital. Estes deveriam pertencer à equipe da UTIN e prestar assistência direta ao RN. Não participaram os profissionais de saúde afastados por qualquer tipo de licença no período da coleta ou que não assinaram o Termo de Consentimento Livre e Esclarecido.

\section{Instrumentos}

Para a caracterização da amostra, foi utilizado um questionário de dados sociodemográficos e 
funcionais sobre a experiência dolorosa pessoal ou familiar, além de um questionário adaptado sobre concepção de prematuro, avaliação e manejo de dor em procedimentos invasivos, especialmente elaborado a partir dos estudos de Porter, Wolf, Gold, Lotsoff e Miller (1997), Souza, Santos, Souza, Gonçalves e Paiva (2006) e Stevens et al. (2011b). Os demais instrumentos foram os seguintes:

1) Effort-Reward Embalance (ERI) - versão curta (Chor, Werneck, Faerstein, Mello, \& Rotenberg, 2008; Siegrist, 1996): com 23 itens que avaliam o estresse ocupacional. Os itens são categorizados em Esforço, Recompensa e Supercomprometimento;

2) Escala de Coping Ocupacional (ECO), de Latack (1986), traduzida e adaptada por Pinheiro, Tróccoli e Tamayo (2003): apresenta 29 itens relacionados à maneira de lidar com problemas do ambiente de trabalho, os quais são distribuídos em três fatores: Controle $(\alpha=0,79)$, Esquiva $(\alpha=0,77)$ e Manejo de Sintomas $(\alpha=0,81)$;

3) Diagnóstico Organizacional (DO) (Krausz, 1994): verifica, em 12 áreas, o grau de permeabilidade a intervenções que objetivam introduzir um processo de mudança planejada: relações interpessoais; padrões de relacionamento; relações intergrupais; padrões de comunicação; canais de comunicação; estilos de liderança; tomada de decisão; planejamento; resolução de problemas; trabalho em equipe; clima organizacional; e motivação;

4) Escala de Clima Organizacional para Organizações de Saúde (ECOOS) (Menezes et al., 2009): a escala foi adaptada para esta pesquisa e contou com 60 itens, quatro a menos que a versão original, isso porque os mesmos não tinham relação com a UTIN. Os itens são organizados em sete fatores: liderança; desenvolvimento profissional; relacionamento e espírito de equipe; relação com a comunidade; segurança no trabalho; estratégias; e remuneração. A análise é baseada em quatro dimensões: Psicossocial, Organizacional, Ocupacional e Extraorganizacional;

5) Protocolo de entrevista, com 16 questões, elaborado a partir dos resultados dos demais instrumentos. As questões foram organizadas em: Con- cepção de prematuro; Dor neonatal; Estresse e Coping ocupacional; e Ambiente da UTIN.

\section{Procedimentos}

Todos os preceitos éticos da legislação para a pesquisa em saúde foram obedecidos, com as devidas aprovações do Comitê de Ética em Pesquisa (CEP) da Universidade Federal do Espírito Santo (UFES) (Processo n 260/11, de 01/03/2012) e CEP do Hospital Infantil Nossa Senhora da Glória (Processo $n^{\circ}$ 01/2012, de 20/03/2012). O estudo foi desenvolvido em quatro etapas, com variação no tamanho da amostra em função do objetivo e disponibilidade no período de coleta (março de 2012 a abril de 2013).

Nesse período, a Secretaria Estadual de Saúde do Estado do Espírito Santo anunciou a desativação da maternidade e sua transferência para outra instituição do mesmo município. A UTIN continuou nas dependências do hospital com a função de assistir aos RN nascidos em outras maternidades do estado e que necessitassem de cuidados intensivos. A despeito dessa situação particular, na Etapa 1 participaram 84 profissionais (21 médicos, 14 enfermeiros e 49 técnicos de enfermagem), correspondendo a $89 \%$ do total de 94 profissionais de saúde do setor. Com essa amostra, foram coletados, por questionários, os dados sobre concepção de prematuro e processo de reconhecimento, avaliação e tratamento da dor durante a realização de 20 procedimentos invasivos de rotina. Paralelamente, foi avaliado se a realização de procedimento invasivo no prematuro era reconhecida como fator estressor na UTIN, bem como a intensidade e o grau de dor neonatal e a prática diária e esperada de analgesia nos 20 procedimentos.

Na Etapa 2, participaram 80 profissionais da Etapa 1, visto que quatro não pertenciam ao quadro da UTIN. Nessa etapa, foram identificados e analisados indicadores de estresse e coping ocupacional no ambiente de trabalho, a partir de escalas validadas e adaptadas para esse fim (ERI e ECO). Na Etapa 3, considerando os instrumentos selecionados (DO e ECOOS), optou-se por realizar uma amostra probabilística $(n=25)$ por sorteio entre cada cate- 
goria profissional da amostra da Etapa 1, tendo sido feito um diagnóstico organizacional e um levantamento sobre o clima organizacional, com o objetivo de analisar seu grau de influência no processo de mudança planejada para o manejo adequado do alívio da dor.

Por fim, na Etapa 4, foram realizadas entrevistas individualizadas com 15 participantes da Etapa 3, pois houve perda da amostra nessa última fase da coleta, incluindo pedido de exoneração de cargo ou término de contrato. Todavia, foi possível ter representantes de cada segmento profissional na amostra final, permitindo, a partir do reconhecimento de variáveis identificadas nas etapas anteriores, uma melhor compreensão das variáveis pessoais e de contexto que possivelmente influenciam a prática no manejo da dor neonatal.

\section{Resultados}

A concepção, avaliação e o manejo da dor neonatal por 84 profissionais de saúde de UTIN, analisados a partir do questionário e da entrevista, mostraram que a concepção de RNPT relacionou-se ao peso de nascimento abaixo de $2.500 \mathrm{~g}$; já a prematuridade foi entendida pelo peso ao nascimento, mas sem relação com a idade gestacional. No questionário, todos os participantes responderam que consideram que o RNPT sente dor. $\mathrm{Na}$ identificação da dor, as principais respostas assinaladas das características comportamentais do bebê foram: choro $(95,2 \%)$, agitação $(92,9 \%)$ e olhos espremidos $(81,0 \%)$; apenas $23,8 \%$ desses profissionais marcaram a quietude do RNPT como um sinal de dor. Em relação ao conhecimento sobre a dor e suas consequências, a maioria $(97,6 \%)$ demonstrou a importância do tratamento da mesma, embora somente 32,1\% disseram ter conhecimento de escala/instrumento para avaliação da dor. Além disso, 14 dos 20 procedimentos médicos invasivos (70,0\%) eram realizados sem analgesia, com exceção daqueles considerados mais dolorosos pelos profissionais. Contudo, quase todos $(97,6 \%)$ responderam haver interferência da dor no desenvolvimento do RNPT.
A maioria dos procedimentos médicos invasivos investigados era realizada sem medidas de alívio da dor, sendo os mais dolorosos a drenagem torácica, punção suprapúbica, intubação orotraqueal, punção lombar e punção de veia/artéria; já os considerados menos dolorosos foram sondagem orogástrica e aspiração oral/nasal. Dos 20 procedimentos avaliados, 14 nunca eram realizados com analgesia e apenas seis foram avaliados como aqueles que sempre deveriam ser realizados com analgesia: drenagem torácica, punção suprapúbica, intubação orotraqueal, punção lombar, punção veia/ artéria e inserção periférica do cateter central.

A aplicação das escalas de estresse ocupacional (ERI) e de coping ocupacional (ECO), em conjunto com os dados de entrevistas com 80 profissionais da UTIN, indicaram que a falta de infraestrutura e de rotina de trabalho, além de comprometimento com a assistência, foram apontadas como os estressores mais comuns na UTIN. Em termos de estresse no trabalho, houve equilíbrio entre esforço e recompensa (Média - $M<1$ ). Houve diferença significativa entre os profissionais apenas no "comprometimento organizacional" ( $p=0,00$; teste de Tukey): médicos $(M=15)$ e enfermeiros $(M=14)$ estavam mais próximos ao risco de estresse ocupacional (valores $\geq 18$ ). A estratégia de enfrentamento mais frequente foi a de "controle" $(M=3,4)$, indicando que esses profissionais tinham uma atitude positiva frente ao estressor, ou seja, uma tentativa de resolução ativa dos problemas referentes ao trabalho na UTIN.

O conjunto de valores, atitudes e padrões de comportamento no ambiente da UTIN, que retrata o grau de satisfação dos profissionais de saúde $(n=25)$, foi medido por sete fatores da ECOOS, sendo caracterizado por: comunicação difícil entre profissionais e demais setores do hospital, dificultando a resolução de problemas; falta de gerenciamento, de estrutura básica de serviço e de padronização na assistência; inexistência de trabalho em equipe; precárias condições de trabalho; e desmotivação. Apenas a dimensão "relacionamento e espírito de equipe" foi um pouco melhor avaliada pelos profissionais (Tabela 1). 
Clima organizacional da Unidade de Terapia Intensiva Neonatal segundo profissionais de saúde $(n=25)$

\begin{tabular}{|c|c|c|c|c|c|c|c|c|c|c|}
\hline \multirow{3}{*}{ Fatores } & \multicolumn{10}{|c|}{ Concordância } \\
\hline & \multicolumn{2}{|c|}{ Não } & \multicolumn{2}{|c|}{ Pouco } & \multicolumn{2}{|c|}{ Razoavelmente } & \multicolumn{2}{|c|}{ Bastante } & \multicolumn{2}{|c|}{ Totalmente } \\
\hline & $\mathrm{n}$ & $\%$ & $n$ & $\%$ & $\mathrm{n}$ & $\%$ & $\mathrm{n}$ & $\%$ & $\mathrm{n}$ & $\%$ \\
\hline \multicolumn{11}{|l|}{ Dimensão Psicossocial } \\
\hline Liderança $\left({ }^{*} n=375\right)$ & 147 & 39,2 & 124 & 33,0 & 74 & 19,7 & 28 & 7,4 & 2 & 0,5 \\
\hline Relacionamento e espírito de equipe $\left({ }^{*} n=197\right)$ & 23 & 11,6 & 48 & 24,3 & 72 & 36,5 & 41 & 20,8 & 13 & 6,5 \\
\hline \multicolumn{11}{|l|}{ Dimensão Ocupacional } \\
\hline Desenvolvimento profissional $\left({ }^{*} n=297\right)$ & 140 & 47,1 & 64 & 21,5 & 45 & 15,1 & 42 & 14,1 & 6 & 2,0 \\
\hline \multicolumn{11}{|l|}{ Dimensão Organizacional } \\
\hline Remuneração $\left({ }^{*} n=95\right)$ & 59 & 62,1 & 20 & 21,0 & 10 & 10,5 & 5 & 5,2 & 1 & 1,0 \\
\hline Segurança $\left({ }^{*} n=120\right)$ & 43 & 35,8 & 31 & 25,8 & 32 & 26,6 & 14 & 11,6 & 0 & 0,0 \\
\hline Estratégias de trabalho $\left({ }^{*} n=191\right)$ & 117 & 61,2 & 41 & 21,4 & 27 & 14,1 & 5 & 2,6 & 1 & 0,5 \\
\hline \multicolumn{11}{|l|}{ Dimensão Extraorganizacional } \\
\hline Relacionamento comunidade $\left({ }^{*} \mathrm{n}=190\right)$ & 59 & 31,0 & 65 & 34,2 & 44 & 23,1 & 20 & 10,5 & 2 & 1,0 \\
\hline Total $=1.479$ & 588 & 39,7 & 393 & 26,5 & 304 & 20,5 & 155 & 10,0 & 39 & 2,6 \\
\hline
\end{tabular}

Nota: *n: número total de respostas na dimensão avaliada; Escala de Clima Organizacional para Organizações de Saúde (ECOOS) (Menezes et al., 2009).

Com base nos resultados, foram identificados fatores que dificultavam a realização do trabalho, podendo influenciar o engajamento dos profissionais de saúde no manejo de dor neonatal. Esses fatores estavam relacionados: (a) às crenças, como a dor ser inerente ao cuidado neonatal; (b) a organização do trabalho, como o desvio de funções; e (c) a capacitação profissional, como falta de protocolos, rotina e treinamentos. Entre os fatores facilitadores, tem-se: (a) as crenças, especialmente sobre o prematuro necessitar de cuidados especiais e sentir dor; (b) a percepção de controle no ambiente de trabalho e a avaliação positiva dos potenciais estressores da UTIN; (c) a organização do trabalho, quando os profissionais se percebem como parte de uma equipe com experiência; e (d) a capacitação profissional, com os indicadores comportamentais da dor.

A partir de uma perspectiva motivacional (Skinner \& Wellborn, 1994), foram reconhecidas algumas relações que podem atuar como fontes de estresse por ameaçarem suas necessidades de relacionamento, competência e autonomia, no contexto da UTIN. Na dimensão organizacional, foram identificadas algumas condições típicas de um contexto de "negligência", como falta de preocupação com a segurança, baixa remuneração e precárias senso de pertencimento, de vinculação ou de relacionamento no contexto do trabalho.

Outros estressores estavam relacionados à condição de "caos" no ambiente da UTIN, caracterizado como "desorganizado", sem rotina e protocolos. Essa situação pode ameaçar o senso de competência profissional, tanto que foi identificada ausência de condições adequadas para prestação da assistência ao RNPT devido à superlotação, falta de materiais e equipamentos.

O estilo de liderança no período avaliado foi considerado autoritário, com tomada de decisão centralizada, caracterizando um contexto de "coerção". Esses estressores ameaçavam a necessidade de autonomia pessoal e no trabalho, como mostraram os dados da avaliação da dimensão psicossocial. Os estressores identificados, contudo, tenderam a ser avaliados pela maioria dos profissionais de saúde do estudo como um "desafio" à sua necessidade de vinculação, de competência e de autonomia, levando-os a lidar de forma adaptativa com as dificuldades do contexto, adotando estratégias de enfrentamento do tipo "controle".

Por fim, o contexto social foi percebido como não fornecedor de "apoio à autonomia", sendo caracterizado por tomada de decisão centralizada e liderança autoritária. É um contexto que não 
favorece a percepção de controle e autonomia, como mostram os dados da dimensão psicossocial, indicando, também, a necessidade de mudanças pela escala DO.

Ainda na análise dos dados dessa escala, o contexto social da UTIN foi percebido pelos profissionais como favorável em termos de "envolvimento", com um bom padrão de relacionamento.
Dessa forma, não havia uma ameaça à sua vinculação com o trabalho e colegas, tanto que a relação interpessoal foi avaliada como "apoiadora" e os padrões de relacionamento como "confiáveis". Outras características do ambiente também foram percebidas como "estruturadas", como a adequada assistência aos pacientes quando em boas condições de trabalho. Estas favorecem a percepção de com-

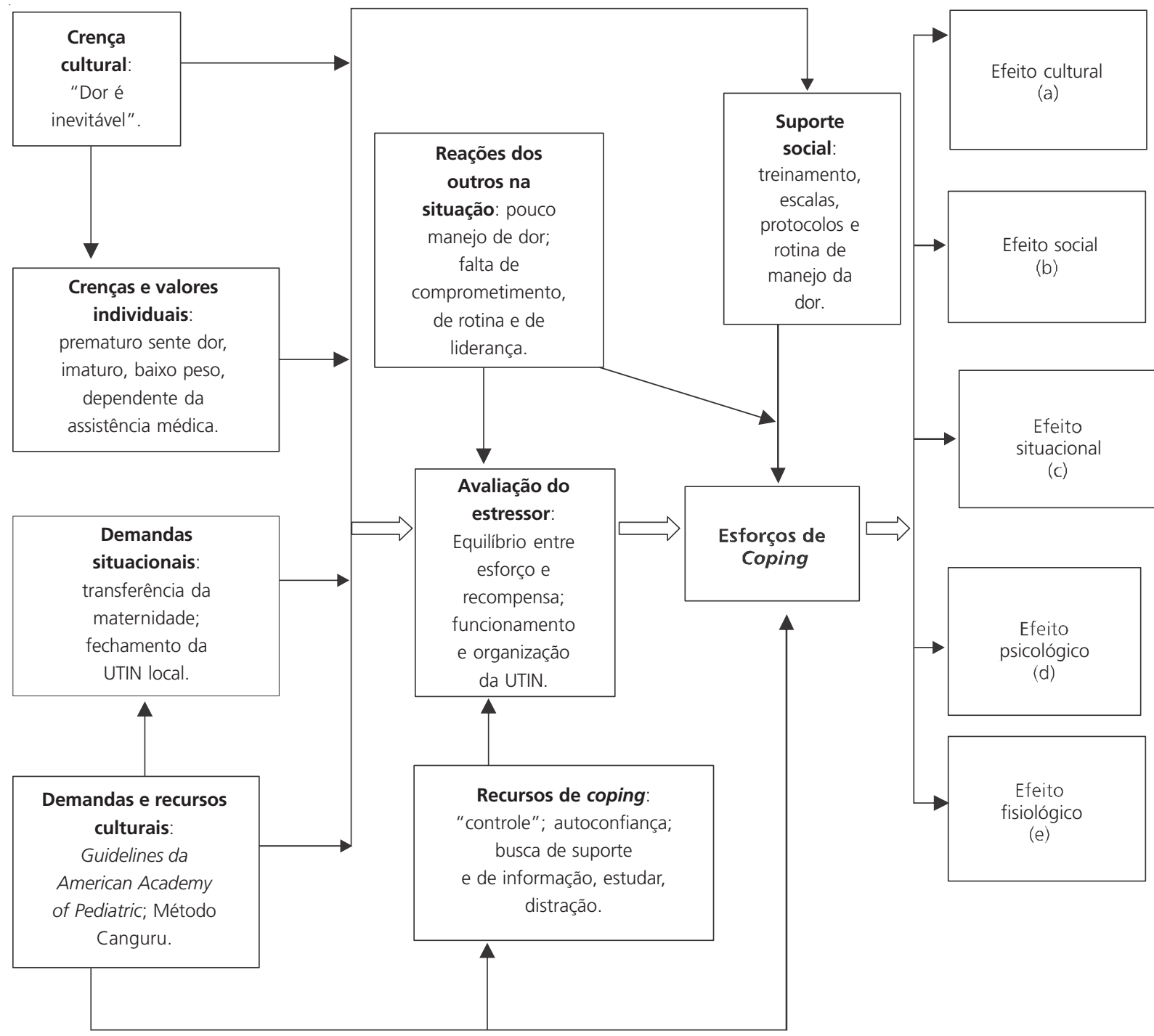

Figura 1. Modelo de relações de variáveis socioculturais de estresse e coping e adaptação dos profissionais de saúde.

Fonte: Aldwin (2009, p.247).

Nota: (a) reafirmação de valores e crenças culturais sobre a capacidade do neonato prematuro sentir dor e suas consequências sobre o desenvolvimento infantil; (b) confirmação e manutenção de valores e crenças individuais sobre a capacidade do prematuro sentir dor e a certeza das consequências no desenvolvimento quando o manejo não é adequado; (c) treinamento e capacitação para manejo de dor, adoção de protocolos e rotina de manejo da dor aguda e crônica; (d) maior engajamento nas atividades profissionais; e (e) ausência de mal-estar físico, estresse fisiológico, poucas faltas e/ou afastamento no trabalho devido a problemas de saúde.

UTIN: Unidades de Terapia Intensiva Neonatal. 
petência, como mostram os dados que indicam ausência de estresse no cuidado direto ao RNPT. Colocando esses dados em um esquema mais amplo, segundo modelo de relações de variáveis socioculturais de estresse e coping e adaptação de Aldwin (2009), que podem favorecer ou não o manejo da dor neonatal, tem-se o quadro representado na Figura 1.

A compreensão coletiva de que a dor é inevitável durante a internação na UTIN tem relação direta com a crença individual de que o prematuro sente dor e depende da assistência prestada, crenças estas apoiadas pela política de atenção à saúde do prematuro e fomentadas pelos manuais do manejo da dor (American Academy of Pediatrics \& Canadian Paediatric Society, 2000; 2006) e pelo Método Canguru (Brasil, 2000). Contudo, as demandas situacionais durante a coleta de dados, representadas pela provável transferência da maternidade e a possibilidade de fechamento da UTIN, afetaram diretamente a avaliação do estresse ocupacional. Nesse caso em particular, contribuiu para uma avaliação negativa, retratada por mal funcionamento e organização da UTIN, falta de rotina e protocolos de avaliação e manejo da dor e, ainda, ausência de treinamento e/ou uso de escalas de dor.

Diante desse quadro, as estratégias de enfrentamento do estresse no trabalho se caracterizaram por tentativa de controle da situação. Os profissionais de saúde apresentaram, em geral, um coping ocupacional que resulta em efeitos mais adaptativos, mantendo sua percepção de controle da situação e evitando o desamparo. Uma postura mais proativa foi identificada na manutenção da crença da capacidade do prematuro de sentir dor, no reconhecimento das consequências para o desenvolvimento infantil e na cobrança de treinamento para o manejo adequado da dor. Como esses são comportamentos mais adaptativos para lidar com o estresse, constataram-se, como consequência, ausência de mal-estar físico e estresse psicológico e nenhum afastamento do trabalho por problemas de saúde.

\section{Discussão}

$\mathrm{Na}$ análise das variáveis que influenciam o 640 processo de engajamento ou não de profissionais de saúde de UTIN no manejo da dor, sob uma perspectiva motivacional (Skinner \& Wellborn, 1994) e sociocultural do coping (Aldwin, 2009), os participantes caracterizaram a prematuridade somente com base no peso de nascimento, abaixo de $2.500 \mathrm{~g}$, diferentemente da definição padrão da World Health Organization (1977). O neonato acima de $2.500 \mathrm{~g}$ não foi considerado prematuro, independentemente da idade gestacional, concepção esta que requer especial cuidado para que a assistência não seja negligenciada, pois, para esses profissionais, a aparência do RN é a de um bebê a termo. Portanto, a concepção de prematuro deve ser considerada na lista dos fatores relacionados ao profissional que podem afetar a prática de manejo da dor infantil (Twycross, Dowden, \& Bruce, 2013).

A noção de que o prematuro não está pronto para nascer e, por esse motivo, necessita do cuidado prestado na UTIN, e de que é capaz de sentir dor durante a assistência, apresentam-se como variáveis facilitadoras na implantação de boas práticas de cuidados, como a da avaliação e tratamento da dor. Já sua sobrevivência está vinculada à responsabilidade da equipe e requer uma quantidade variável de procedimentos dolorosos necessários. A concordância, para a maioria, de que o prematuro é capaz de sentir dor, corrobora a mudança de paradigma no que tange à percepção da dor pediátrica pelos profissionais de saúde, principalmente da dor do RNPT (American Academy of Pediatrics \& Canadian Paediatric Society, 2000, 2006).

Assim como no trabalho de Gibbins et al. (2015), os profissionais deste estudo reconheceram a existência da dor neonatal, principalmente pelos parâmetros comportamentais (mímica facial e choro). Contudo, só uma minoria apontou a quietude do RN como sinal de dor, indicando que a ausência do choro dificulta o uso de medidas de alívio. $\mathrm{Na}$ implantação dessas medidas, a identificação de dor em um neonato quieto significa uma medida protetora do ambiente, mas requer do profissional uma observação mais rigorosa das reações do bebê, deixando de lado a ideia de que a dor está apenas relacionada com agitação e/ou choro.

Os profissionais deste estudo tinham conhecimento das consequências da dor repetida e 
prolongada no período neonatal, referindo a importância de seu manejo devido à possível interferência no desenvolvimento, acarretando transtornos cognitivos ou emocionais. Reconhecer que a dor traz consequências para o futuro do neonato pode ser um fator facilitador para o engajamento dos profissionais na implantação da analgesia em suas práticas.

Apesar do reconhecimento dos efeitos deletérios, os participantes afirmaram que as medidas de alívio da dor não eram realizadas com a frequência desejada. A execução dos procedimentos invasivos, na maioria das vezes, era feita sem qualquer analgesia, com exceção da drenagem torácica. Esses resultados corroboram conclusões de outros estudos sobre as dificuldades para a realização dessas práticas na rotina da UTIN pela equipe de saúde (Martins et al., 2013a; Porter et al., 1997).

Dos 20 procedimentos avaliados, 14 nunca eram realizados com analgesia. Essa baixa frequência foi justificada devido às condições de trabalho na UTIN, as quais não favoreciam um cuidado adequado ao manejo da dor baseado em evidências científicas. Os profissionais estavam preocupados com a execução das tarefas e relataram ausência de cobrança da chefia quanto ao manejo, a falta de educação continuada na área da dor neonatal, assim como a falta de protocolos e de rotinas. Alguns desses resultados são semelhantes aos encontrados no estudo de Linhares et al. (2014) sobre a dor pediátrica.

A constatação de que a analgesia efetiva e adequada não fazia parte da prática diária dos profissionais de UTIN reafirma o fato de que somente o reconhecimento da dor e de suas consequências no desenvolvimento não é suficiente para produzir mudança de atitude diante da dor do neonato (Aymar et al., 2014). Como considerado, variáveis as quais envolvem a adesão ao manejo da dor neonatal estão relacionadas às concepções do profissional de saúde sobre se e como o prematuro sente dor e a necessidade de usar medidas de alívio da mesma, mas outra parte da explicação pode estar no próprio contexto de trabalho.

Para a execução de boas práticas no cuidado ao RN, incluindo manejo de dor adequado e efi- ciente, é importante a avaliação da organização da UTIN (Stevens et al., 2011a). Este estudo mostrou que a variável organizacional tem influência marcante na atuação profissional, dificultando a execução das medidas de alívio da dor nos procedimentos invasivos de rotina na UTIN.

Na presente pesquisa, o clima organizacional da UTIN foi avaliado como caótico, com profissionais insatisfeitos. Essa avaliação negativa pode ter sido influenciada, em parte, pelo momento difícil em que os profissionais se encontravam no período de coleta de dados na UTIN, devido ao provável fechamento do setor e redistribuição da equipe para outros hospitais. Nesse sentido, um clima organizacional potencialmente negativo pode gerar estresse no profissional de saúde, somando-se à própria condição de trabalho na UTIN, que se caracteriza por um ambiente fechado, com rotinas exigentes e extenuantes e questões éticas delicadas, além do convívio constante com o sofrimento, a morte e a imprevisibilidade.

Dados obtidos pela escala ERI (Chor et al., 2008), contudo, não indicaram maior sinal de estresse, pois houve equilíbrio entre esforço e recompensa no trabalho, sem evidências de supercomprometimento. Esses dados diferem do estudo de Fogaça, Carvalho, Cítero e Nogueira-Martins (2010) com médicos e enfermeiros de uma UTIN, que identificou um desequilíbrio no enfrentamento desses profissionais. Ambos os estudos, porém, se assemelharam quanto à tendência, na categoria de médicos, ao supercomprometimento, enfatizando a importância da detecção de fatores de riscos ocupacionais.

Dados qualitativos evidenciaram que não era a assistência direta ao RNPT que causava estresse, mas sim fatores relacionados à infraestrutura de trabalho, à assistência, aos recursos humanos e ao comprometimento profissional. Em outras palavras, as dificuldades em oferecer assistência adequada devido às condições ruins de trabalho eram compreendidas como um desserviço à população pediátrica.

Embora o contexto fosse estressante, de insatisfação e receio dos profissionais devido à transferência da UTIN e à ausência de uma política de investimento para manutenção da unidade exis- 
tente, a estratégia de enfrentamento de "manter o controle" foi prevalente na amostra. O tipo de estratégia de enfrentamento adotada demonstrou que os profissionais buscavam alternativas resolutivas, ativas e diretas, no âmbito individual e ambiental, na tentativa de modificar o estressor, visando desempenhar as funções sob sua responsabilidade de maneira mais adaptativa. Mais especificamente, as estratégias de enfrentamento de autoconfiança, busca de suporte social, busca de informação e resolução de problemas foram predominantes na amostra. A percepção de controle das ações para lidar com a fonte geradora de estresse no trabalho da UTIN, associada à reavaliação cognitiva positiva, pode ter influenciado o comportamento de pedir ajuda para lidar com o problema, como observado nos relatos os quais exemplificam as categorias "busca de suporte social" e "resolução de problemas".

A adoção de estratégias de enfrentamento de controle reduz o relato de ansiedade relacionada ao trabalho e sua associação positiva com o suporte social se deve ao fato de que este último tem um papel maior do que simples apoio emocional (Latack, 1986). Houve um engajamento dos indivíduos em estratégias proativas diante de situações de estresse. Mesmo diante da precária situação de funcionamento da UTIN, principal causadora do estresse ocupacional, os profissionais apresentaram reações e respostas ativas, positivas e direcionadas ao estressor (Skinner \& Wellborn, 1994). Esse tipo de coping adaptativo indica que, se o estressor ambiente for modificado, no caso, a organização e o funcionamento caótico da UTIN, existirá a possibilidade de maior comprometimento do profissional e, com isso, a implantação das boas práticas de assistência ao RNPT, com a probabilidade de avaliação e tratamento adequados da dor neonatal.

O modelo sociocultural de estresse, coping e adaptação (Aldwin, 2009) permitiu melhor compreensão sobre como o contexto cultural afeta a maneira do profissional de saúde lidar com o estresse do trabalho, por identificar os fatores da cultura organizacional, os quais podem influenciar suas práticas. Essa identificação viabiliza a proposição

642 de estratégias específicas, que possam culminar com a mudança necessária de comportamento para que medidas de alívio de dor sejam implantadas de forma efetiva no ambiente da UTIN.

A importância do fator organizacional influenciando o comportamento do profissional, no que se refere ao engajamento ou não em práticas adequadas e efetivas de assistência, demonstrou a complexidade que envolve o trabalho em uma UTIN. Julgamentos sobre o modo de se comportar da equipe não têm valor se não forem analisados sob a ótica da relação entre outras variáveis, além da díade profissional-paciente. Quanto à implantação de medidas de alívio da dor, esta será possível a partir da compreensão das influências de cada variável investigada e de outras que não foram aqui apontadas, como a assistência centrada na família e a avaliação dos pais quanto ao manejo da dor, possibilitando uma discussão multidisciplinar cujo objetivo final é a melhoria da assistência aos neonatos.

Os resultados indicaram que o alívio da dor em uma Unidade Neonatal, para ser adequado e efetivo, requer muito mais que implantação de escalas e protocolos de tratamento. A importância de escalas de dor e de uma rotina de manejo, principalmente na dor aguda, certamente não é questionada. Contudo, se faz necessária uma análise mais ampla do ambiente da UTIN, visando identificar as variáveis que possam facilitar ou dificultar a mudança de comportamento diante da dor neonatal e, assim, a elaboração de propostas de implantação de medidas de avaliação e tratamento.

Houve limitações de caráter metodológico, como, por exemplo, o número variável de participantes ao longo das etapas da pesquisa, uma possível interferência na coleta, pois uma das pesquisadoras que conduziu as entrevistas pertencia à própria equipe de profissionais investigados, e a situação diferenciada da UTIN selecionada (antes da transferência). Contudo, este estudo poderá servir como modelo no intuito de sistematizar as etapas de investigação de importantes variáveis associadas à dor neonatal e sua implicação na rotina profissional.

A prática de medidas de alívio da dor na UTIN requer uma minuciosa pesquisa, já que sua concretização depende de vários fatores inerentes 
ao paciente, ao profissional, ao ambiente e também aos familiares, que atuam diretamente no tipo de assistência oferecida. O conhecimento científico sobre a dor e suas consequências para o neonato prematuro, a experiência profissional e a adoção de protocolos e rotinas de manejo da dor baseados em evidências não garantem uma mudança de comportamento diante da dor neonatal que culmine com alívio adequado e efetivo no período de internação na UTIN. A dor tem relação direta com variáveis culturais, sociais, ambientais, psicológicas e educacionais e todas necessitam ser pelo menos discutidas quando se propõe a elaboração de protocolos e rotinas para o manejo da dor no ambiente hospitalar e, principalmente, na Unidade Neonatal.

As informações obtidas poderão ser úteis aos setores responsáveis pela humanização do hospital por abordar algumas variáveis do ambiente, visando à capacitação profissional na aplicação de boas práticas de assistência neonatal, como ocorre no manejo da dor. Sem uma cuidadosa avaliação das concepções sobre o neonato e sua dor, das práticas de alívio em procedimentos invasivos, dos estressores do ambiente de trabalho e coping ocupacional, qualquer tentativa de implantação de protocolos e rotina de manejo de dor poderá ser infrutífera, pois essas variáveis atuam diretamente na mudança do cenário de uma UTIN.

\section{Colaboradores}

S.W. MARTINS colaborou na concepção e desenho, análise e interpretação dos dados, e discussão dos resultados. S.R.F. ENUMO contribuiu na concepção e desenho, análise e interpretação dos dados, discussão dos resultados e revisão e aprovação da versão final do artigo. K.M.P. PAULA colaborou na análise e interpretação dos dados, discussão dos resultados, e revisão e aprovação da versão final do artigo.

\section{Referências}

Aldwin, C. M. (2009). Sociocultural aspects of coping. In C. M. Aldwin. Stress, coping, and development: An integrative perspective (pp.239-270). New York: The Guilford Press.
American Academy of Pediatrics, \& Canadian Paediatric Society. (2000). Prevention and management of pain and stress in the neonate. Pediatrics, 105(2), 454-461.

American Academy of Pediatrics, \& Canadian Paediatric Society. (2006). Prevention and management of pain in the neonate: An update. Pediatrics, 118(5), 2231-2241. http://dx.doi.org/10.1542/peds.2006-2277

Anand, K. J. S., Stevens, B. J., \& McGrath, P. J. (2007). Pain in neonates and infants. London: Elsevier.

Aymar, C. L. G., Lima, L. S., Santos, C. M. R., Moreno, E. A. C., \& Coutinho, S. B. (2014). Pain assessment and management in the NICU: Analysis of an educational intervention for health professional. Jornal de Pediatria, 90(3), 308-315. http://dx.doi.org/10.1016/j. jped.2013.09.008

Bispo, C. A. (2006). Um novo modelo de pesquisa de clima organizacional. Produção, 16(2), 258-273. http:// dx.doi.org/10.1590/S0103-65132006000200007

Brasil. Ministério da Saúde. (2000). Norma de atenção humanizada do recém-nascido de baixo peso - Método Canguru. Brasília: Autor.

Brummelte, S., Grunau, R. E., Chaw, V., Poskitt, K. J., Vinall, J., Gover, A., ... Miller, S. P. (2012). Procedural pain and brain development in premature infant. Annals Neurology, 71(3), 385-396. http://dx.doi.org/ 10.1002/ana.22267

Chor, D., Werneck, G. L., Faerstein, E., Mello, M. C. A., \& Rotenberg, L. (2008). The Brazilian version of ERI to assess job stress. Caderno de Saúde Pública, 24(1), 219-224.

Fogaça, M. C., Carvalho, W. B., Cítero, V., \& Nogueira-Martins, L. A. (2010). Estudo preliminar sobre o estresse ocupacional de médicos e enfermeiros em UTI pediátrica e neonatal: o equilíbrio entre esforço e recompensa. Revista Latino-Americana de Enfermagem, 18(1), 67-72. http://dx.doi.org/10:1590/\$0104-1169 20110000100011

Gibbins, S., Stevens, B., Dionne, K., Yamada, J., Riddell, R. P., McGrath, ... Johnston, C. (2015). Perceptions of health professional on pain in extremely low gestational age infants. Qualitative Health Research, 25(1), 763-774. http//dx.doi.org/10.1177/104972315 580558

Grunau, R. E. (2013). Neonatal pain in very preterm infants: Long-term effects on brain, neurodevelopment and pain reactivity. Rambam Maimonides Medical Journal, 4(4), 1-13. http://dx.doi.org/10.5041/RMMJ. 10132

Hall, W. (2012). Anesthesia and analgesia in the NICU. Clinics in Perinatology, 39(1), 239-254. http://dx.doi. org/10.1016/j.clp.2011.12.013

Krausz, R. R. (1994). Diagnóstico organizacional: Forma I e Forma II - Manual de aplicação e interpretação. São Paulo: Casa do Psicólogo. 
Latack, J. C. (1986). Coping with job stress: Measures and future directions for scale development. Journal of Applied Psychology, 71(3), 377-385. http://dx.doi. org/10.1037/0021-9010.71.3.377

Linhares, M. B. M., Doca, F. N. P., Martinez, F. E., Carlotti, A. A. P., Cassiano, R. R. M., Pfeifer, L. I., ... Finley, G. A. (2012). Pediatric pain: Prevalence, assessment, and management in a teaching hospital. Brazilian Journal of Medical and Biological Research, 45(12), 1287-1294. http://dx.doi.org/10.1590/S0100-879X20120075 00147

Linhares, M. B. M., Oliveira, N. C. A. C., Doca, F. N. P., Martinez, F. E., Carlotti, A. P. P., \& Finley, G. A. (2014). Assessment and management of pediatric pain based on the opinions of health professional. Psychology \& Neuroscience, 7(1), 43-53. http://dx.doi.org/10.3922/j. psns.2014.1.07

Martins, S. W., Dias, F. S., Paula, K. M. P., \& Enumo, S. R. F. (2013a). Pain assessment and control by nurses of a Neonatal Intensive Unit. Revista Dor, 14(1), 21-26. http://dx.doi.org/10.1590/S1806-0013201300010 0006

Martins, S. W., Cruzeiro, M. S., Paula, K. M. P., \& Enumo, S. R. F. (2013b). Clima organizacional e sua influência no manejo da dor neonatal. In A. Garcia, M. D. C. Macedo, \& T. A. Nunes (Orgs.), Relações interpessoais e saúde (pp.154-164). Vitória: GM Gráfica e Editora.

McPherson, C., Haslam, M., Pineda, R., Rogers, C., Neill, J. J., \& Inder, T. E. (2015). Brain injury and development in preterm infants exposed to fentanyl. Annals Pharmacotherapy, 49(12), 1291-1297. http://dx.doi. org/10.1177/1060028015606732

Menezes, I. G., Sampaio, L. R., Gomes, A. C. P., Teixeira, F. S., \& Santos, P. S. (2009). Escala de clima organizacional para organizações de saúde: desenvolvimento e estrutura fatorial. Estudos de Psicologia (Campinas), 26(3), 305-316. http://dx.doi.org/10.15 90/S0103-166X2009000300004

Montali, L., Monica, C., Riva, P., \& Cipriani, R. (2011). Conflicting representations of pain: A qualitative analysis of health care professionals' discourse. Pain Medicine, 12(11), 1585-1593. http://dx.doi.org/10. 1111/j.1526-4637.2011.01252.X

Passini Junior, R., Cecatti, J. G., Lagos, G. L., Tedesco, R. P., Nomura, M. L., Dias, T. Z., ... Souza, M. H. (2014). Brazilian Multicentre Study on Preterm Birth (EMIP): Prevalence and factors associated with spontaneous preterm birth. Plos One, 9(10), e109069.

Pinheiro, F. A., Tróccoli, B. T., \& Tamayo, M. R. (2003). Mensuração de coping no ambiente ocupacional. Psicologia: Teoria e Pesquisa, 19(2), 153-158.

Porter, F. L., Wolf, C. M., Gold, J., Lotsoff, D., \& Miller, J. P. (1997). Pain and pain management in newborn infants: A survey of physicians and nurses. Pediatrics, 100(4), 626-632. http://dx.doi.org/10.1542/peds. 10 0.4 .626

Ramos, F. P., Enumo, S. R. F., \& Paula, K. M. P. (2015). Teoria Motivacional do Coping: uma proposta desen- volvimentista de análise do enfrentamento do estresse. Estudos de Psicologia (Campinas), 32(2), 269-279. http://dx.doi.org/10.1590/0103-166X201500020 0011

Ramos, F. P., Enumo, S. R. F., Paula, K. M. P., \& Vicente, S. C. R. M. (2010). Concepções de funcionários de UTIN sobre competências desenvolvimentais de recémnascidos. Psicologia: Teoria e Prática, 12(2), 144-157.

Shonkoff, J. P., Ricther, L., Gaag, J., \& Bhutta, Z. A. (2012). An integrated scientific framework for child survival and early childhood development. Pediatrics, 129, 460-471. http://dx.doi.org/10.1542/peds.2011-0366

Siegrist, J. (1996). Adverse health effects of high-effort/ low-reward conditions. Journal of Occupational Health Psychology, 1(1), 27-41. http://dx.doi.org/10.1037/10 76-8998.1.1.27

Silva, D. A., \& Marcolan, J. F. (2015). Desemprego e sofrimento psíquico em enfermeiras. Revista Brasileira Enfermagem, 68(5), 775-785. http://dx.doi.org/10.15 90/0034-7167.201580502i

Skinner, E. A., \& Wellborn, J. G. (1994). Coping during childhood and adolescence: A motivational perspective. In P. B. Baltes \& O. G. Brim (Eds.), Life span development and behavior (pp.91-133). Hillsdale: L. Relbaum.

Souza, B. B. B., Santos, M. H., Souza, F. G. M., Gonçalves, A. P. F., \& Paiva, S. S. (2006). Avaliação da dor como instrumento para o cuidar de recém-nascidos pré-termo. Texto \& Contexto Enfermagem, 15(Esp.), 88-96.

Stevens, B. J., Riahi, S., Cardoso, R., Ballantyne, M., Yamada, J., Beyene, J., \& Ohlsson, A. (2011a). The influence of context on pain practices on NICU: Perceptions of health care professionals. Qualitative Health Research, 21(6), 757-770. http://dx.doi.org/ 10.1177/1049732311400628

Stevens, B. J., Abbott, L. K., Yamada, J., Harrison, D., Stinson, J., Taddio, A., ... Finley, G. A. (2011b). Epidemiology and management of painful procedures in children in Canadian hospitals. Canadian Medical Association Journal, 183(7), 183-187. http://dx.doi.org/10.1503/ cmaj.101341

Theme Filha, M. M., Costa, M. A. S., \& Guilam, M. C. R. (2013). Estresse ocupacional e auto avaliação de saúde entre profissionais de enfermagem. Revista Latino-Americana de Enfermagem, 21(2), 475-483. http:// dx.doi.org/10.1590/S0104-11692013000200002

Twycross, A., Dowden, S., \& Bruce E. (2013). Managing pain in children: A clinical guide for nurses and healthcare professionals. New Jersey: Wiley-Blackwell.

World Health Organization. (1977). Recommended definitions, terminology and format for statistical tables related to the perinatal period and use of a new certificate for cause perinatal deaths. Acta Obstetricia et Gynecologica Scandinavica, 56(3), 247-253.

Recebido: janeiro 31, 2016

Versão final: fevereiro 9, 2016

Aprovado: fevereiro 12, 2016 